\title{
Association between Platelet Counts before and during Pharmacological Therapy for Patent Ductus Arteriosus and Treatment Failure in Preterm Infants
}

\section{OPEN ACCESS}

Edited by:

Eugene Dempsey,

University College Cork, Ireland

Reviewed by:

Joseph Ting,

University of British

Columbia, Canada

Karel Allegaert,

University Hospitals Leuven,

Belgium

*Correspondence:

Hannes Sallmon

hannes.sallmon@charite.de

Specialty section:

This article was submitted

to Neonatology,

a section of the journal

Frontiers in Pediatrics

Received: 07 November 2017

Accepted: 12 February 2018

Published: 07 March 2018

Citation:

Sallmon H, Weber SC, Dirks J, Schiffer T, Klippstein T, Stein A,

Felderhoff-Müser U, Metze B, Hansmann G, Bührer C, Cremer M and Koehne $P$ (2018) Association between Platelet Counts before and during Pharmacological Therapy for

Patent Ductus Arteriosus and Treatment Failure in Preterm Infants.

Front. Pediatr. 6:41.

doi: 10.3389/fped.2018.00041

\begin{abstract}
Hannes Sallmon ${ }^{1 *}$, Sven C. Weber ${ }^{2}$, Juliane Dirks', Tamara Schiffer', Tamara Klippstein ${ }^{1}$, Anja Stein ${ }^{3}$, Ursula Felderhoff-Müser ${ }^{3}$, Boris Metze ${ }^{1}$, Georg Hansmann ${ }^{4}$, Christoph Bührer ${ }^{1}$, Malte Cremer ${ }^{1}$ and Petra Koehne ${ }^{1}$

${ }^{1}$ Department of Neonatology, Charité University Medical Center, Berlin, Germany, ${ }^{2}$ Department of Pediatrics, Neonatology and Pediatric Intensive Care Medicine, University of Greifswald, Greifswald, Germany, ${ }^{3}$ Division of Neonatology, Department of Pediatrics I, University Hospital Essen, Essen, Germany, ${ }^{4}$ Department of Pediatric Cardiology and Critical Care, Hannover Medical School, Hannover, Germany
\end{abstract}

Background: The role of platelets for mediating closure of the ductus arteriosus in human preterm infants is controversial. Especially, the effect of low platelet counts on pharmacological treatment failure is still unclear.

Methods: In this retrospective study of 471 preterm infants [ $<1,500 \mathrm{~g}$ birth weight (BW)], who were treated for a patent ductus arteriosus (PDA) with indomethacin or ibuprofen, we investigated whether platelet counts before or during pharmacological treatment had an impact on the successful closure of a hemodynamically significant PDA. The effects of other factors, such as sepsis, preeclampsia, gestational age, BW, and gender, were also evaluated.

Results: Platelet counts before initiation of pharmacological PDA treatment did not differ between infants with later treatment success or failure. However, we found significant associations between low platelet counts during pharmacological PDA therapy and treatment failure $(p<0.05)$. Receiver operating characteristic $(R O C)$ curve analysis showed that platelet counts after the first, and before and after the second cyclooxygenase inhibitor (COXI) cycle were significantly associated with treatment failure (area under the curve of $>0.6$ ). However, ROC curve analysis did not reveal a specific platelet cutoff-value that could predict PDA treatment failure. Multivariate logistic regression analysis showed that lower platelet counts, a lower BW, and preeclampsia were independently associated with COXI treatment failure.

Conclusion: We provide further evidence for an association between low platelet counts during pharmacological therapy for symptomatic PDA and treatment failure, while platelet counts before initiation of therapy did not affect treatment outcome.

Keywords: preterm infant, ibuprofen, indomethacin, patent ductus arteriosus, platelets, ductal closure

Abbreviations: BW, birth weight; CBC, complete blood count; COX, cyclooxygenase; COXI, cyclooxygenase inhibitor; CRIB, clinical risk indek for babies; DA, ductus arteriosus; DOL, days of (postnatal) life; ELBW, extremely low birth weight infant; GA, gestational age; PDA, patent ductus arteriosus; hsPDA, hemodynamically significant PDA; NICU, neonatal intensive care unit; NSAID, non-steroidal anti-inflammatory drug; ROC, receiver operating characteristic; VLBW, very low birth weight infant. 


\section{INTRODUCTION}

Ductus arteriosus (DA) closure is an important step during the transition from fetal to extrauterine neonatal life. Failure of spontaneous DA closure in immature infants can be associated with several complications such as left ventricular volume overload, pulmonary edema, and impairment of lung compliance (1). However, due to a high spontaneous closure rate and a favorable outcome of infants who were discharged home with a persistently patent DA, it is controversial which patent ductus arteriosus (PDAs) require treatment (2). The aforementioned associations between adverse outcomes and a large and significant PDA provide the rationale for many clinicians to initiate treatment for hemodynamically significant PDAs (hsPDA) (3). The current mainstay of therapy is the administration of the cyclooxygenase (COX) inhibitors ibuprofen or indomethacin. These drugs act by inhibiting COX involved in producing prostaglandins E1 and E2 that are known to promote ductal patency. The success rate of this pharmacological approach, however, varies and is difficult to predict for individual infants $(1,3)$.

In mice, platelet-triggered ductal sealing and subsequent vascular remodeling appears to be an important mechanism for definite DA closure (4). Murine platelets are recruited to the endothelium of the DA soon after birth, and platelet dysfunction or defective platelet formation was found to result in persistently patent DA. The role of platelets for DA closure in humans, however, remains controversial, especially in preterm infants. An association between low platelet counts on the first day of life and PDA has been described in several retrospective analyses (4-7). However, similar analyses failed to confirm these findings (8-13). Two recent meta-analyses indicated weak, but significant, associations between hsPDA and thrombocytopenia on the first day of life and pre-treatment thrombocytopenia and pharmacological treatment failure, respectively $(14,15)$.

However, data from these studies on platelet counts during and just before initiation of therapy by day of life 4 or later are sparse in the reported investigations $(15,16)$. We herein analyzed whether platelet counts before initiation and during pharmacological PDA therapy are associated with treatment failure in a large cohort of very low birth weight infant (VLBW) infants.

\section{MATERIALS AND METHODS}

\section{Patients}

This retrospective cohort study was conducted at the neonatal intensive care units (NICUs) in the Department of Neonatology, Campus Virchow-Klinikum and Campus Mitte, Charité University Medical Center, Berlin Germany (1998-2008). All VLBW infants born in the respective period were included if they had received pharmacological treatment for hsPDA. Platelet counts before initiation of therapy, after the first, and before and after a second or third treatment cycle (if administered) were recorded, and the incidence of treatment success in the different groups was calculated. The clinical risk index for babies (CRIB) was calculated and parental consent for data collection was obtained after patient admission to the NICUs.

\section{PDA Diagnosis/Algorithm for PDA Intervention}

Infants were examined for hsPDA on days of (postnatal) life (DOL) 4-5 and when clinically indicated. Echocardiographic evaluation included shunt direction of a PDA in color Doppler mode (high upper parasternal short axis) as well as the minimal internal ductal diameter of three to five measurements taken in B-mode. The left atrium to aortic root (LA/Ao) ratio was measured by M-mode (parasternal long axis). The Doppler measurement of the resistance index in the anterior cerebral artery was done at the same time. Examinations were performed as previously described (8).

Cyclooxygenase inhibitor (COXI) treatment was initiated in all VLBW infants with an hsPDA. A PDA with left-to-right shunt was considered hemodynamically significant if (i) a respiratory set back with a supplemental oxygen requirement $>30 \%$ and/or mechanical ventilation, (ii) a LA/Ao ratio $\geq 1.4$ in the echocardiogram and/or (iii) ductal diameter $\geq 2.5 \mathrm{~mm}$, and/or (iv) a decreased end-diastolic flow in the anterior cerebral artery with a resistance index $\geq 0.85$ in the cerebral ultrasound were present. There has been no substantial change in the clinical and echocardiographic criteria used to determine the need for PDA intervention during the study period.

\section{COXI Therapy}

None of the infants received treatment before an echocardiogram was performed. Infants received indomethacin or ibuprofen $\left(\right.$ Pedea $\left.^{\circledR}\right)$ exactly as previously described in detail (including dosing, duration, etc.) (8). Successful response to COXI treatment was defined as absent ductal shunt flow $24-48 \mathrm{~h}$ after therapy, all other cases were defined as COXI treatment failure. Echocardiographic reassessment was performed after each therapy cycle.

\section{Statistics}

Normality was tested by Kolmogorov-Smirnov test. Comparisons between groups were made by Kruskal-Wallis or Mann-Whitney $U$-test as appropriate for continuously scaled data and by chisquare test for categorical data. $p$ Values $<0.05$ were considered statistically significant. Statistical analyses, including multivariate logistic regression and receiver operating characteristic (ROC) curve analyses, were carried out using the IBM SPSS Statistics 19 software.

\section{RESULTS}

\section{Incidence of PDA and Treatment Success Rates}

Out of a total of 1,641 VLBW infants, we included 471 patients (28.7\%) who were treated with COXI for hsPDA in the respective period (including 353 extremely low birth weight infant infants, Figure 1). Demographics of the patient cohort are shown in Table 1. The first COXI course was permanently successful in $48.8 \%(230 / 471)$ of all VLBW infants. Of note, although 332 infants (70.5\%) achieved initial DA closure after the first COXI course, 102 infants developed reopening of their PDA after the first treatment course. Out of the remaining 241 infants, 


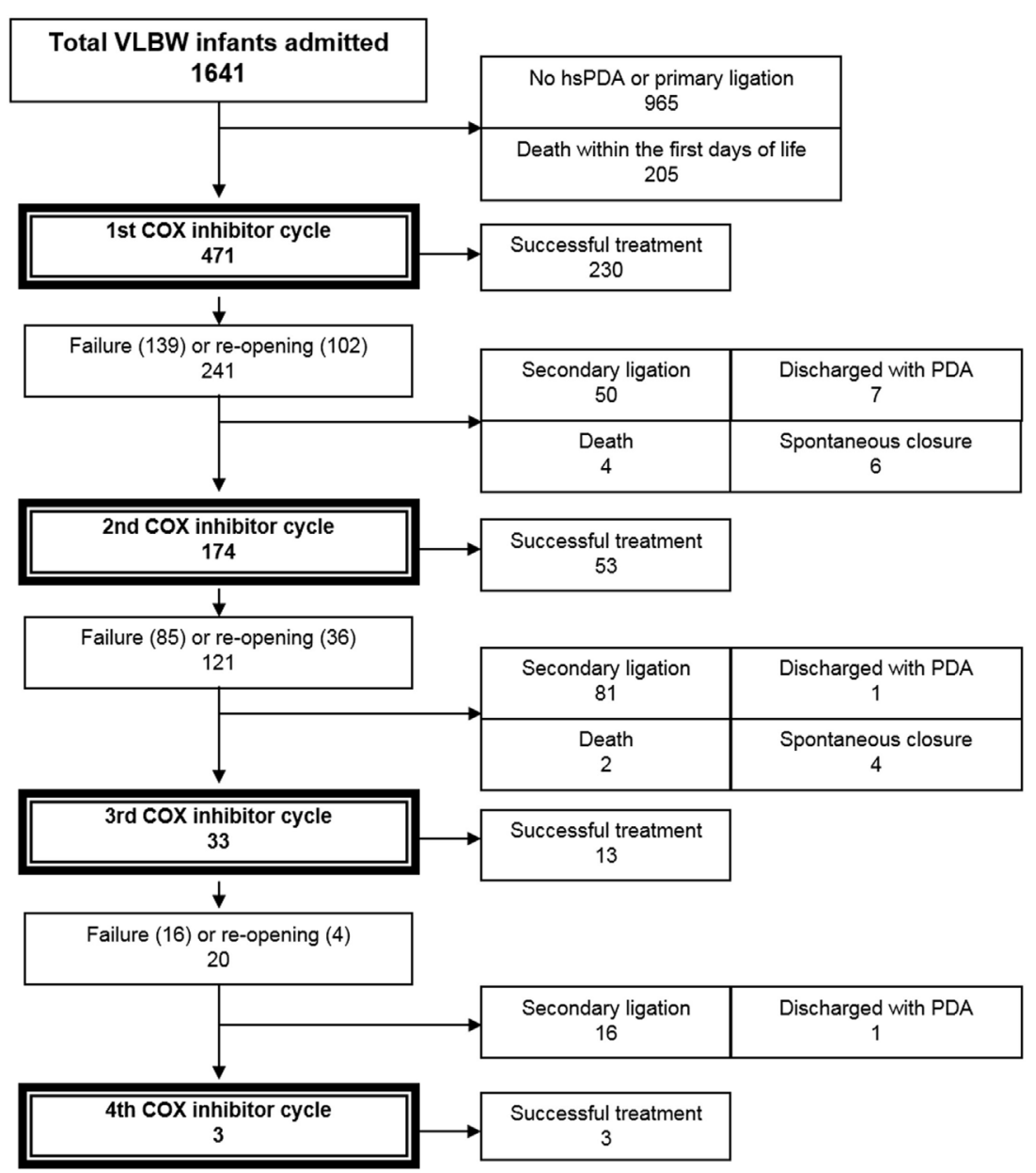

FIGURE 1 | Flowchart: study population. Treatment with COX inhibitors was considered successful, if no further PDA therapy was required. COX, cyclooxygenase; hsPDA, hemodynamically significant PDA; PDA, patent ductus arteriosus; VLBW, very low birth weight $(<1,500 \mathrm{~g})$.

TABLE 1 | Characteristics of VLBW infants undergoing pharmacological treatment by cyclooxygenase inhibitors (COXI) for hemodynamically significant patent ductus arteriosus.

\begin{tabular}{lccc}
\hline VLBW infants & COXI Total & COXI Success & COXI Failure \\
\hline$N(\%)$ & $471(100)$ & $299(63.5)$ & $172(36.5)$ \\
Female $(n, \%)$ & $208(44)$ & $123(41.1)$ & $85(49.4)$ \\
Birth weight (BW) (g) & $867(270-1.490)$ & $875(442-1.490)$ & $\mathbf{7 4 9}(\mathbf{2 7 0 - 1 . 4 8 0})^{\star *}$ \\
Gestational age & $26(23-34)$ & $26(23-34)$ & $\mathbf{2 5}\left(\mathbf{2 3 - 3 4 ) ^ { \star * }}\right.$ \\
(weeks) & & & \\
Twins $(n, \%)$ & $125(26.5)$ & $78(26.1)$ & $47(27.3)$ \\
Triplets $(n, \%)$ & $25(5.3)$ & $16(5.4)$ & $9(5.2)$ \\
Quadruplets $(n, \%)$ & $3(0.6)$ & $1(0.3)$ & $2(1.2)$ \\
Apgar 5 min & $7(1-9)$ & $7(1-9)$ & $7(1-9)$ \\
CRIB score & $6(0-17)$ & $5(0-17)$ & $\mathbf{7}(\mathbf{0 - 1 7})^{\star *}$ \\
Preeclampsia $(n, \%)$ & $60(12.7)$ & $45(15.1)$ & $\mathbf{1 5}(\mathbf{8 . 7})$ \\
Sepsis $(n, \%)$ & $417(88.5)$ & $253(84.6)$ & $\mathbf{1 6 4}(\mathbf{9 5 . 3})$ \\
\hline
\end{tabular}

Data are numbers (percentages) or median (range). In the top row, data in parentheses represent percentages referring to the total number of 471 included VLBW preterm infants (horizontal comparison within the first row). For all other data shown, percentages in parentheses refer to the total number in the upper first row of the same column (vertical comparison within the same column). Statistically significant values are given in bold $\left({ }^{*} p<0.05 ;{ }^{* *} p<0.001\right)$.

VLBW, very low birth weight infant $(<1,500 \mathrm{~g})$; COXI, cyclooxygenase inhibitor (indomethacin or ibuprofen); CRIB, clinical risk index for babies.
174 received a second, 33 a third, and 3 a fourth COXI course (Figure 1). In total, 299/471 infants (63.5\%) achieved permanent ductal closure by pharmacological treatment, while 147 (31.2\%) infants underwent secondary ligation. Nine patients (1.9\%) were discharged home with a hsPDA, $6(1.3 \%)$ died, and $10(2.1 \%)$ experienced spontaneous PDA closure before a subsequent treatment cycle was initiated. Of note, treatment success rates between the infants that initially received indomethacin or ibuprofen were not significantly different $(p>0.05)$.

\section{Platelet Counts before Initiation and during Therapy and Treatment Success Rates}

The platelet counts before initial pharmacological treatment did not differ significantly between infants with and infants without successful treatment. However, the first platelet counts after completion of the first COXI course differed significantly between infants with initial treatment success and failure $(p<0.001)$. Similar results were obtained for the platelet counts just before $(p<0.05)$ and after the second COXI cycle $(p<0.05$, Table 2). Trends toward higher platelet counts in the treatment 
TABLE 2 | Platelet numbers in very low birth weight infants treated for hemodynamically significant PDA before and after each course of cyclooxygenase inhibitors.

\begin{tabular}{|c|c|c|c|}
\hline & COXI success & COXI failure & Time point (day of life) \\
\hline \multicolumn{4}{|c|}{ Platelet counts before 1st COXI cycle } \\
\hline Median & 201,000 & 196,000 & 3 \\
\hline Minimum & 27,000 & 11,000 & 1 \\
\hline Maximum & 608,000 & 531,000 & 52 \\
\hline \multicolumn{4}{|c|}{ Platelet counts after 1st COXI cycle } \\
\hline Median & 308,000 & $220,000^{\star *}$ & 11 \\
\hline Minimum & 19,000 & 35,000 & 3 \\
\hline Maximum & 812,000 & 633,000 & 60 \\
\hline \multicolumn{4}{|c|}{ Platelet counts before 2nd COXI cycle } \\
\hline Median & 309,000 & $233,000 *$ & 14 \\
\hline Minimum & 56,000 & 12,000 & 4 \\
\hline Maximum & 580,000 & 497,000 & 63 \\
\hline \multicolumn{4}{|c|}{ Platelet counts after 2nd COXI cycle } \\
\hline Median & 289,000 & $198,000^{*}$ & 19 \\
\hline Minimum & 31,000 & 24,000 & 7 \\
\hline Maximum & 598,000 & 634,000 & 52 \\
\hline \multicolumn{4}{|c|}{ Platelet counts before 3rd COXI cycle } \\
\hline Median & 354,000 & 200,000 & 20 \\
\hline Minimum & 96,000 & 13,000 & 10 \\
\hline Maximum & 631,000 & 502,000 & 66 \\
\hline \multicolumn{4}{|c|}{ Platelet counts after 3rd COXI cycle } \\
\hline Median & 328,500 & 181,000 & 30 \\
\hline Minimum & 105,000 & 30,000 & 14 \\
\hline
\end{tabular}

Platelet counts are given in number/ $\mu L$. Statistically significant differences between treatment success and failure are given in bold $\left({ }^{*} p<0.05 ;{ }^{* *} p<0.001\right)$. COXI, cyclooxygenase inhibitor.

success group were also seen before and after the third course of COXI treatment. However, these results did not reach statistical significance due to a lower $n$ number.

\section{ROC Curve and Logistic Regression Analyses}

Next, we performed a ROC curve analysis which showed a significant association between platelet counts after the first COXI cycle and treatment failure with an area under the curve (AUC) of 0.617 and a confidence interval (CI) of 0.560-0.674. However, the form of the curve indicated no specific cutoff that could be used to predict treatment failure below a specific platelet count (Figure 2). Of note, similar results were obtained for platelet counts before and after the second COXI cycle (data not shown).

In this study, preeclampsia was associated with significantly lower platelet counts at birth $(p<0.001)$. Similarly, infants with sepsis, defined by elevated systemic markers of inflammation (IL-6 and/or CRP) and the use of antibiotics, showed a nonsignificant trend toward lower platelet counts before initiation of therapy. Multivariate logistic regression analysis showed that higher platelet counts after the first COXI cycle were independently associated with treatment success, while sepsis, and gender had no significant effect (Table 3). In addition, birth weight (BW) [or gestational age (GA)], and preeclampsia were also independent predictors of treatment success rates.

\section{DISCUSSION}

The role of platelets during closure of the DA is subject to ongoing debates. While experimental data suggest that platelets are involved in spontaneous ductal closure (4), the clinical implications of these findings are controversial especially in very immature infants. In our previous study on 1,350 VLBW infants, we found no association between platelet counts within the first $24 \mathrm{~h}$ after birth and spontaneous ductal closure. We also did not find an association between platelet count on DOL 1 and a later treatment failure. However, sepsis, male gender, a lower BW, or GA were risk factors for hsPDA development (8). Similarly, Shah et al. assessed serial platelet counts (DOL $1-7$ ) in preterm infants born $<28$ weeks of gestation and PDA closure rates $(n=497)$. Neither high nor low platelet numbers had an impact on permanent DA closure. However, in this study, all infants received prophylactic indomethacin starting on DOL 1 (17). Data on the platelet counts during and just before initiation of symptomatic PDA therapy by day of life 4 or later are sparse in the reported investigations $(15,16)$.

In this study, we addressed this issue by investigating serial platelet counts in relation to PDA treatment success, which provides a differential picture on the influence of platelet counts before and after each COXI cycle and its success. We were able to demonstrate, that platelet counts just before initiation of pharmacological PDA therapy (median DOL 3) were not associated with treatment failure. However, we found statistically significant associations between platelet counts during COXI therapy and the respective success rates. Furthermore, a lower BW, a lower GA, and a higher CRIB score were significantly associated with treatment failure of COXI therapy in this cohort. Interestingly, male gender, which others and we previously reported to be an independent risk factor for development of an hsPDA, was not significantly associated with treatment failure rates. Similarly, preeclampsia, which is known to affect platelet numbers $(18,19)$, was significantly associated with COXI therapy failure. Interestingly, sepsis/infection had no effect on treatment success rates, which is likely due to the less strict definition used in this study (elevated systemic markers of infection, use of antibiotics).

The available data on platelet counts and their implications for DA closure and PDA treatment success are controversial. The reported heterogeneity of previous studies might be explained by different definitions of hsPDA, different echocardiographic screening protocols (parameters and time points), and by different treatment protocols (prophylactic vs. symptomatic treatment). This study cannot definitively answer the question of how clinically relevant low platelet counts are for ductal closure in human preterm infants. However, it provides evidence for an association between low platelet counts during COXI therapy initiated between DOL 3 and 5 for symptomatic PDA and treatment failure. Logistic regression analysis showed a significant albeit weak independent effect of platelet counts during PDA therapy on success rates. These findings currently do not change clinical practice as no clear cutoff value that was associated with treatment failure could be established.

Besides platelet numbers, thrombocyte function might play a role during DA closure. The most immature infants are also 


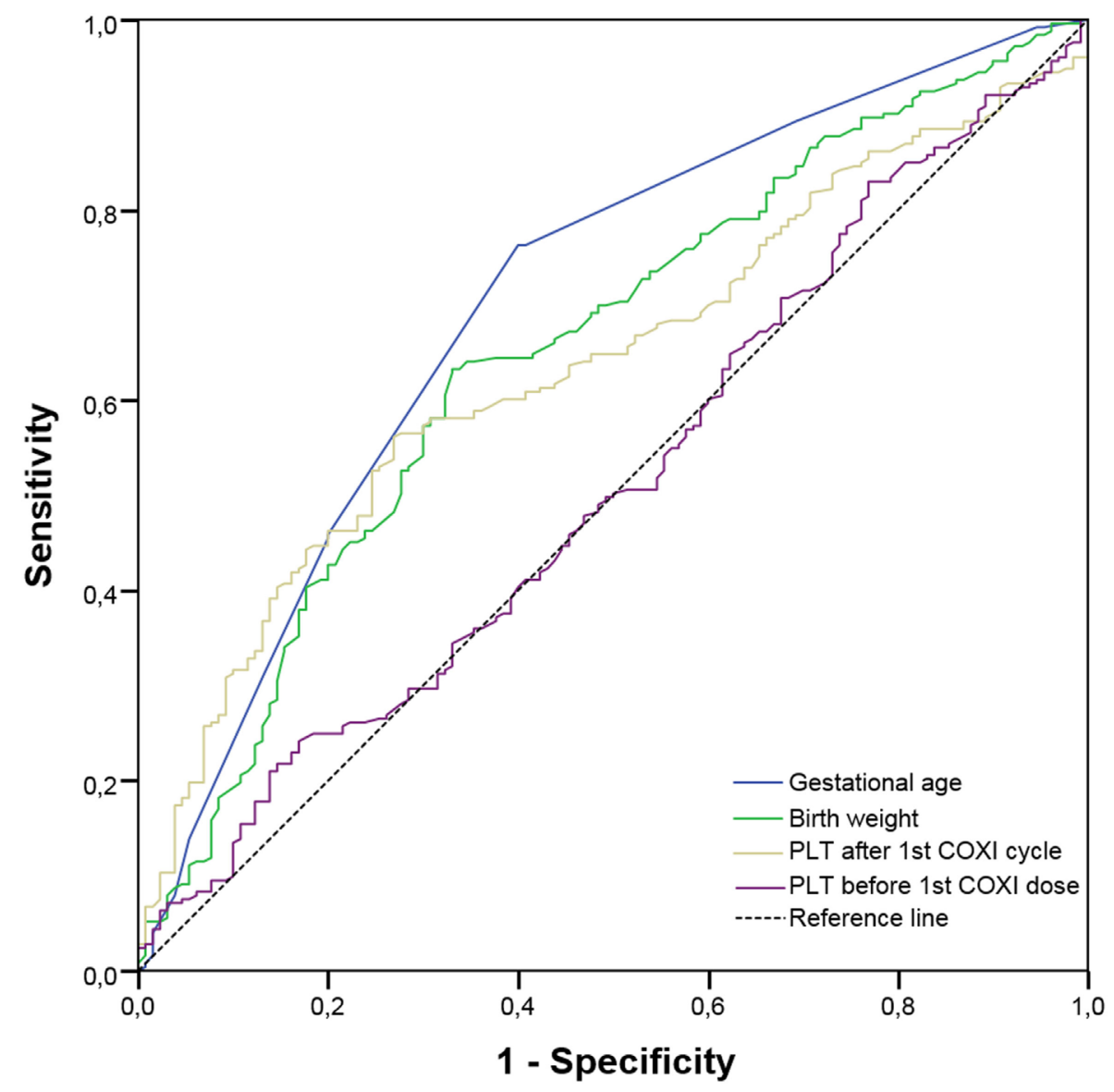

FIGURE 2 | Receiver operating characteristic (ROC) curve analysis. The ROC curve analysis shows a significant association between platelet counts after the first cyclooxygenase inhibitor (COXI) cycle and treatment failure with an area under the curve (AUC) of 0.617 and a confidence interval (CI) of 0.560-0.674. However, the form of the curve indicated no specific cutoff that could be used to predict treatment failure below a specific platelet count. Of note, similar results were obtained for platelet counts before and after the second COXI cycle (data not shown). For comparison, we also included platelet counts before the first COXI dose (AUC 0.513;

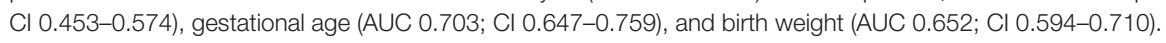

TABLE 3 | Logistic regression analysis for prediction of COXI treatment success.

\begin{tabular}{|c|c|c|c|c|c|c|}
\hline & $B$ & SE & Wald & df & Significance & $\operatorname{Exp}(B)$ \\
\hline Gestational age $e^{\star \star \star}$ & 0.294 & 0.073 & 16.140 & 1 & $<0.001$ & 1.342 \\
\hline $\begin{array}{l}\text { PLT after 1st COXI } \\
\text { cycle }^{\star \star \star}\end{array}$ & 0.003 & 0.001 & 12.629 & 1 & $<0.001$ & 1.003 \\
\hline Preeclampsia* & -0.867 & 0.414 & 4.379 & 1 & 0.036 & 0.420 \\
\hline Sepsis/infection & 0.315 & 0.464 & 0.460 & 1 & 0.497 & 1.370 \\
\hline Male gender & -0.306 & 0.237 & 1.669 & 1 & 0.196 & 0.736 \\
\hline Constant & -0.922 & 1.967 & 12.380 & 1 & 0.000 & 0.001 \\
\hline
\end{tabular}

$381 / 471$ (80.9\%) of cases with a complete dataset were included in this analysis. Of note, sepsis/infection was defined by elevated markers of infection and antibiotic therapy. Significant factors $\left({ }^{*} p<0.05,{ }^{* * *} p<0.001\right)$ are listed in bold.

$B$, coefficient for the constant; Wald, Wald chi-square test; $d$, degree of freedom;

$\operatorname{Exp}(B)$, exponent $B ; P L T$, platelet number; COXI, cyclooxygenase inhibitor.

most likely to have impaired platelet function (e.g., due to sepsis, immaturity, or medication). Therefore, future prospective investigations should include parameters of platelet function (20), especially in infants receiving non-steroidal anti-inflammatory drugs (NSAIDs), which are known to potentially alter platelet function.
In regard to this, Kahvecioglu et al. reported longer collagen-ADP times in infants with hsPDA (21). Other authors have suggested that parameters such as platelet mass or mean platelet volume might be important for DA closure $(6,11,12,21,22)$.

NSAIDs are standard of care in closing a hsPDA in preterm infants (3). Overall, we observed successful ductal closure after initial therapy in $63.5 \%$ of all VLBW infants, respectively, which is consistent with the ranges reported in the literature (1). In the patients analyzed, NSAIDs were usually withheld if platelet counts were $<50,000 / \mu \mathrm{L}$. A total of 55 infants received platelet transfusions during COXI treatment, which did not influence the treatment success rates. NSAID treatment of infants with moderate thrombocytopenia $(50,000-100,000 / \mu \mathrm{L})$ was considered save. However, this assumption was based on the only randomized controlled trial on platelet transfusion thresholds performed to date. Andrew et al. randomized 152 thrombocytopenic infants to receive platelet transfusions for any platelet count $<150,000 / \mu \mathrm{L}$ or for a platelet count $<50,000 / \mu \mathrm{L}$ (or clinical indications) targeting a primary outcome of IVH. These investigators found no significant differences in frequency or severity of IVH between the 
two groups (23). However, Brunner et al. reported an increased risk for intracerebral bleeding in VLBW infants with moderate thrombocytopenia that were treated with COXI (24).

These findings illustrate the critical need for well-designed prospective randomized trials that investigate the outcome of thrombocytopenic neonates and the safety of different platelet transfusion thresholds $(19,25,26)$. Furthermore, trials investigating the optimal PDA management (indication for treatment, optimal dosing of NSAIDs, etc.) are essential to improve outcomes, of the most immature infants.

\section{ETHICS STATEMENT}

This study was carried out in accordance with the recommendations of the local ethics committee at Charité Berlin. Formal ethics approval for this specific study was not required due to the retrospective nature of the investigations.

\section{REFERENCES}

1. Hamrick SE, Hansmann G. Patent ductus arteriosus of the preterm infant. Pediatrics (2010) 125(5):1020-30. doi:10.1542/peds.2009-3506

2. Weber SC, Weiss K, Buhrer C, Hansmann G, Koehne P, Sallmon H. Natural history of patent ductus arteriosus in very low birth weight infants after discharge. J Pediatr (2015) 167(5):1149-51. doi:10.1016/j.jpeds.2015.06.032

3. Sallmon H, Koehne P, Hansmann G. Recent advances in the treatment of preterm newborn infants with patent ductus arteriosus. Clin Perinatol (2016) 43:113-29. doi:10.1016/j.clp.2015.11.008

4. Echtler K, Stark K, Lorenz M, Kerstan S, Walch A, Jennen L, et al. Platelets contribute to postnatal occlusion of the ductus arteriosus. Nat Med (2010) 16(1):75-82. doi: $10.1038 / \mathrm{nm} .2060$

5. Alyamac Dizdar E, Ozdemir R, Sari FN, Yurttutan S, Gokmen T, Erdeve O, et al. Low platelet count is associated with ductus arteriosus patency in preterm newborns. Early Hum Dev (2012) 88(10):813-6. doi:10.1016/j.earlhumdev. 2012.05.007

6. Dani C, Poggi C, Fontanelli G. Relationship between platelet count and volume and spontaneous and pharmacological closure of ductus arteriosus in preterm infants. Am J Perinatol (2013) 30(5):359-64. doi:10.1055/s-0032-1324702

7. Kulkarni VV, Dutta S, Sundaram V, Saini SS. Preterm thrombocytopenia and delay of ductus arteriosus closure. Pediatrics (2016) 138(4):e20161627. doi:10.1542/peds.2016-1627

8. Sallmon H, Weber SC, Huning B, Stein A, Horn PA, Metze BC, et al. Thrombocytopenia in the first 24 hours after birth and incidence of patent ductus arteriosus. Pediatrics (2012) 130(3):e623-30. doi:10.1542/ peds.2012-0499

9. Murphy DP, Lee HC, Payton KS, Powers RJ. Platelet count and associated morbidities in VLBW infants with pharmacologically treated patent ductus arteriosus. J Matern Fetal Neonatal Med (2016) 29(13):2045-8. doi:10.3109/ 14767058.2015.1076785

10. Bas-Suarez MP, Gonzalez-Luis GE, Saavedra P, Villamor E. Platelet counts in the first seven days of life and patent ductus arteriosus in preterm very low-birthweight infants. Neonatology (2014) 106(3):188-94. doi:10.1159/000362432

11. Akar S, Karadag N, Gokmen Yildirim T, Toptan HH, Dincer E, Tuten A, et al. Does platelet mass influence the effectiveness of ibuprofen treatment for patent ductus arteriosus in preterm infants? J Matern Fetal Neonatal Med (2016) 29(23):3786-9. doi:10.3109/14767058.2016.1145207

12. Demir N, Peker E, Ece I, Agengin K, Bulan KA, Tuncer O. Is platelet mass a more significant indicator than platelet count of closure of patent ductus arteriosus? J Matern Fetal Neonatal Med (2016) 29(12):1915-8. doi:10.3109/ 14767058.2015 .1067296

13. Fujioka K, Morioka I, Miwa A, Morikawa S, Shibata A, Yokoyama N, et al. Does thrombocytopenia contribute to patent ductus arteriosus? Nat Med (2011) 17(1):29-30. doi:10.1038/nm0111-29

\section{AUTHOR CONTRIBUTIONS}

HS, GH, and PK conceptualized the study. HS, SW, PK, TK, JD, and TS collected the data. HS, SW, BM, PK, JD, and GH analyzed the data. HS wrote the first draft of the manuscript. All authors contributed to the interpretation of the data and critically reviewed and contributed to the final draft of the manuscript.

\section{FUNDING}

The study was supported by a Lydia-Rabinowitsch-Scholarship (Charité University Medical Center Berlin to PK). We acknowledge support from the German Research Foundation (DFG) and the Open Access Publication Fund of CharitéUniversitätsmedizin Berlin.

14. Simon SR, van Zogchel L, Bas-Suarez MP, Cavallaro G, Clyman RI, Villamor E. Platelet counts and patent ductus arteriosus in preterm infants: a systematic review and meta-analysis. Neonatology (2015) 108(2):143-51. doi: $10.1159 / 000431281$

15. Mitra S, Chan AK, Paes BA; Thrombosis and Hemostasis in Newborns (THIN) Group. The association of platelets with failed patent ductus arteriosus closure after a primary course of indomethacin or ibuprofen: a systematic review and meta-analysis. J Matern Fetal Neonatal Med (2017) 30(2):127-33. doi:10.310 9/14767058.2016.1163684

16. Ahamed MF, Verma P, Lee S, Vega M, Wang D, Kim M, et al. Predictors of successful closure of patent ductus arteriosus with indomethacin. J Perinatol (2015) 35(9):729-34. doi:10.1038/jp.2015.33

17. Shah NA, Hills NK, Waleh N, McCurnin D, Seidner S, Chemtob S, et al. Relationship between circulating platelet counts and ductus arteriosus patency after indomethacin treatment. J Pediatr (2011) 158(6):e1-2. doi:10.1016/j. jpeds.2010.11.018

18. Sola-Visner M, Sallmon H, Brown R. New insights into the mechanisms of nonimmune thrombocytopenia in neonates. Semin Perinatol (2009) 33(1):43-51. doi:10.1053/j.semperi.2008.10.008

19. Sallmon H, Sola-Visner M. Clinical and research issues in neonatal anemia and thrombocytopenia. Curr Opin Pediatr (2012) 24(1):16-22. doi:10.1097/ MOP.0b013e32834ee5cc

20. Sallmon H, Weber SC, von Gise A, Koehne P, Hansmann G. Ductal closure in neonates: a developmental perspective on platelet-endothelial interactions. Blood Coagul Fibrinolysis (2011) 22(3):242-4. doi:10.1097/ MBC.0b013e328344c5ed

21. Kahvecioglu D, Erdeve O, Akduman H, Ucar T, Alan S, Çakır U, et al. Influence of platelet count, platelet mass index, and platelet function on the spontaneous closure of ductus arteriosus in the prematurity. Pediatr Neonatol (2017) 59(1):53-7. doi:10.1016/j.pedneo.2017.01.006

22. Olukman O, Ozdemir R, Karadeniz C, Calkavur S, Mese T, Vergin C. Is there a relationship between platelet parameters and patency of ductus arteriosus in preterm infants? Blood Coagul Fibrinolysis (2017) 28(1):8-13. doi:10.1097/ MBC.0000000000000520

23. Andrew M, Vegh P, Caco C, Kirpalani H, Jefferies A, Ohlsson A, et al. A randomized, controlled trial of platelet transfusions in thrombocytopenic premature infants. J Pediatr (1993) 123(2):285-91. doi:10.1016/S0022-3476 (05)81705-6

24. Brunner B, Hoeck M, Schermer E, Streif W, Kiechl-Kohlendorfer U. Patent ductus arteriosus, low platelets, cyclooxygenase inhibitors, and intraventricular hemorrhage in very low birth weight preterm infants. J Pediatr (2013) 163(1):23-8. doi:10.1016/j.jpeds.2012.12.035

25. Stanworth SJ. Thrombocytopenia, bleeding, and use of platelet transfusions in sick neonates. Hematology Am Soc Hematol Educ Program (2012) 2012:512-6. doi:10.1182/asheducation-2012.1.512 
26. Cremer M, Sallmon H, Kling PJ, Bührer C, Dame C. Thrombocytopenia and platelet transfusion in the neonate. Semin Fetal Neonatal Med (2016) 21(1):10-8. doi:10.1016/j.siny.2015.11.001

Conflict of Interest Statement: The authors declare that the research was conducted in the absence of any commercial or financial relationships that could be construed as a potential conflict of interest. The authors have no financial relationships relevant to this article to disclose.
Copyright $\odot 2018$ Sallmon, Weber, Dirks, Schiffer, Klippstein, Stein, FelderhoffMüser, Metze, Hansmann, Bührer, Cremer and Koehne. This is an open-access article distributed under the terms of the Creative Commons Attribution License (CC BY). The use, distribution or reproduction in other forums is permitted, provided the original author(s) and the copyright owner are credited and that the original publication in this journal is cited, in accordance with accepted academic practice. No use, distribution or reproduction is permitted which does not comply with these terms. 